EPJ Web of Conferences 64, 08004 (2014)

DOI: $10.1051 /$ epjconf/ 20146408004

(C) Owned by the authors, published by EDP Sciences, 2014

\title{
Dynamic Young Stars and their Disks: A Temporal View of NGC 2264 with Spitzer and CoRoT ${ }^{\star}$
}

\author{
Ann Marie Cody ${ }^{1, a}$, John Stauffer ${ }^{1}$, Jérôme Bouvier ${ }^{2}$, and The CSI Team ${ }^{3}$ \\ ${ }^{1}$ Spitzer Science Center, California Institute of Technology, 1200 E California Blvd., Pasadena, CA, USA \\ ${ }^{2}$ UJF-Grenoble 1 / CNRS-INSU, Institut de Planétologie et d'Astrophysique de Grenoble (IPAG) UMR 5274, \\ Grenoble, F-38041, France \\ ${ }^{3}$ http://csi2264.ipac.caltech.edu
}

\begin{abstract}
Variability is a signature feature of young stars. Among the well known light curve phenomena are periodic variations attributed to surface spots and irregular changes associated with accretion or circumstellar disk material. While decades of photometric monitoring have provided a framework for classifying young star variability, we still know surprisingly little about its underlying mechanisms and connections to the surrounding disks. In the past few years, dedicated photometric monitoring campaigns from the ground and space have revolutionized our view of young stars in the time domain. We present a selection of optical and infrared time series from several recent campaigns, highlighting the Coordinated Synoptic Investigation of NGC 2264 ("CSI 2264")- a joint 30-day effort with the Spitzer, CoRoT, and MOST telescopes. The extraordinary photometric precision, high cadence, and long time baseline of these observations is now enabling correlation of variability properties at very different wavelengths, corresponding to locations from the stellar surface to the inner $0.1 \mathrm{AU}$ of the disk. We present some results of the CSI 2264 program, including new classes of optical/infrared behavior. Further efforts to tie observed variability features to physical models will provide insights into the inner disk environment at a time when planet formation may be underway.
\end{abstract}

\section{Introduction}

The end stages of star formation and the beginning of planet formation coincide at approximately one million years (Myr) after the initial conglomeration of molecular cloud cores. Key to the outcome of both processes are the fundamentals of gas accretion onto the star, as well as the structure and dynamics of the surrounding circumstellar disk. The current paradigm for understanding accreting young stars involves gas flowing from the disk along magnetic field lines. It then collides with the stellar surface to produce shocks and hot spots with temperatures in excess of 10,000 K. The disk itself is truncated at a distance of $0.1 \mathrm{AU}$, its inner reaches emitting at near- and mid-infrared wavelengths. In addition to controlling the flow of material onto the central star, magnetic fields may regulate

\footnotetext{
${ }^{\star}$ Based on data from the Spitzer and CoRoT missions. The CoRoT space mission was developed and is operated by the French space agency CNES, with participation of ESA's RSSD and Science Programmes, Austria, Belgium, Brazil, Germany, and Spain.

ae-mail: amc@ipac.caltech.edu
}

This is an Open Access article distributed under the terms of the Creative Commons Attribution License 2.0, which permits unrestricted use, distribution, and reproduction in any medium, provided the original work is properly cited. 
the structure of the inner disk. While promising, this primarily static theoretical picture may omit potentially key physics. For example, recent studies of circumstellar disks have offered evidence of both structural asymmetries and dynamic changes of the inner disk wall ([9], [11]). Current telescopes are unable to spatially resolve the inner AU of the circumstellar environment, and therefore indirect observations are required to probe this region.

Photometric variability- a signature feature of young disk bearing stars- bears the imprint of circumstellar and magnetospheric accretion processes. It has been known for decades (e.g., [13]) that the light curves of $\mathrm{T}$ Tauri stars fluctuate with amplitudes of up to one magnitude on timescales from hours to years. Numerous campaigns have sought to tie flux changes to physical processes (e.g., [12], [4], [19]). Because different wavelengths correspond to distinct regions on the star and in the disk, multiwavelength photometric monitoring offers the prospect of studying the location dependence of variability. Recently, a proliferation of photometric campaigns in the X-ray through infrared has produced thousands of high quality young stellar object (YSO) light curves (e.g., [5], [1], [16]). These culminated with the Coordinated Synoptic Investigation of NGC 2264 (CSI 2264; [6],[7]), a simultaneous effort with a dozen ground and space-based telescopes in the optical, near-infrared, and mid-infrared. Whereas previous studies (e.g., [15], [14]) typically monitored YSOs in one or two filters with sparse ground-based coverage, we now have the opportunity to compare photometric behavior in multiple bands at high precision, assessing the degree of correlation and associated color changes. We present here a collection of initial results of CSI 2264, focusing on the combination of light curves from simultaneous CoRoT optical observations and Warm Spitzer/IRAC monitoring.

\section{The CSI 2264 Monitoring Campaign}

Starting in December 2011, the CSI 2264 campaign performed monitoring of several thousand YSOs in the NGC 2264 cluster, which lies at a distance of approximately $760 \mathrm{pc}$ ([17]). We have conducted a literature search for known members ([21], [18], [8],) and identified approximately 1500 objects from the substellar regime through 7 solar masses, in an approximately one square degree field surrounding the Cone Nebula and central nebulosity. The focus of the campaign were observations by the Warm Spitzer Space Telescope ([23]), in tandem with the CoRoT satellite ([2]). The fields of view for each of these telescopes are shown in Figure 1. While CoRoT observed a single 4 square degree field, Spitzer/IRAC operated in mapping mode, observing successive 5 arcminute fields to cover a 45 arcminute wide region. In addition, Spitzer operated in staring mode for a portion of the observations, pointing at two smaller fields (see Figure 1) for up to 24 hours straight while acquiring high precision (i.e., several millimagnitudes) photometry. For much of September 2011, CoRoT and Spitzer were joined by the MOST space telescope ([22]), along with the Chandra X-ray Observatory and 12 groundbased telescopes. The space-based observations are summarized in Table 1.

The unprecedented aspects of CSI 2264 are its high precision (1\% or better for typical photometric points), high cadence ( 2 hours per IRAC datapoint and $<10$ minutes per CoRoT point), and long

Table 1. Summary of the space telescope observations comprising the CSI 2264 campaign.

\begin{tabular}{cccc}
\hline Telescope & Instrument & Bands & Dates \\
\hline Spitzer & IRAC/mapping & $3.6 \mu \mathrm{m}, 4.5 \mu \mathrm{m}$ & Dec. 3, 2011-Jan. 1, 2012 \\
Spitzer & IRAC/staring & $3.6 \mu \mathrm{m}, 4.5 \mu \mathrm{m}$ & Dec. 3; Dec. 5-6,; Dec. 7-8; Dec. 8-9, 2011 \\
CoRoT & E2 CCD & $3000-10000 \AA$ & Dec. 1, 2011-Jan. 3, 2012 \\
MOST & Science CCD & $3500-7500 \AA$ & Dec. 5, 2011-Jan. 14, 2012 \\
Chandra & ACIS-I & $0.5-8 \mathrm{keV}$ & Dec. 3, 2011-Dec. 9, 2011 \\
\hline
\end{tabular}




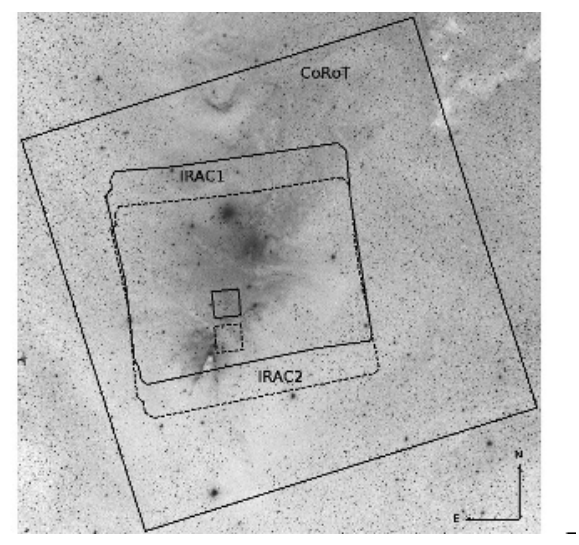

Figure 1. Field of view for Spitzer and CoRoT observations of NGC 2264 as part of the CSI 2264 campaign, overlaid on a Digitized Sky Survey image. The two small boxes representing the fields of staring observations in Spitzer/IRAC channel 1 (solid line) and channel 2 (dashed line). The CoRoT field of view is approximately 1.3 degrees across.

time baseline (30 days for Spitzer and 40 days for CoRoT). NGC 2264 members had been observed previously by both instruments ([1],[16]), but not simultaneously. The combination of precision and time sampling has opened a new view of light curve morphologies.

\section{Light Curve Classification Scheme}

Previous efforts to classify light curves and connect morphology to physical processes associated with YSOs have focused on periodic and aperiodic categories of behavior (e.g., [12] and [1]). The exquisite data quality of the CSI 2264 campaign enables us to classify our light curves on a finer level. We have followed an approach of visually examining and categorizing the light curves first, subsequently confirming our decisions with statistics. A total of eight different morphologies emerge from the set of optical and infrared light curves, and we classify behavior in each band separately. One of the most prominent sets of morphologies involves quasi-periodic fading events that become redder as they grow fainter (see Figure 2). We refer to these as "quasi-periodic dippers" and note that they are the closest analogs to AA Tau (e.g., [3]). A similar morphology, which we call "aperiodic dippers," involves fading events with no detectable periodicity, as shown in Figure 3 (see also [16]). Also displaying fading behavior but in a strictly periodic manner are the eclipsing binaries of NGC 2264. The most intriguing of these exhibits aperiodic out-of-eclipse variability indicative of a circumbinary disk ([10]).

While the dippers and eclipsing binaries display fading, the opposite behavior appears in stars that we refer to as "bursters"- objects with frequent episodes of rapidly increasing flux. This behavior is associated with indicators of strong accretion, such as ultraviolet excess; we highlight it in [20]. The bursters and dippers exhibit what we refer to as asymmetric flux behavior; these light curves appear different when flipped upside-down. Another set of light curves does not have this distinction; we call those with no detectable periodicity "stochastic." For light curves that do show periodicity but are not dippers because their fluxes are symmetric about the time axis, we have chosen the label "quasiperiodic symmetric." The term "periodic" is reserved for objects with the most stable periodicities; this behavior is usually attributed to rotational modulation of cool spots on the stellar surface.

In some cases, a trend is evident in the light curve but it is too featureless to classify; we refer to these as "long timescale" variables. Finally, light curves that are variable but show no behavior matching the descriptions above are left unclassified. We have evaluated a set of 162 disk-bearing YSO light curves with both CoRoT and Spitzer data, and we present the results in Table 2. Stars that show no brightness fluctuations as measured by the light curve root mean square or a Stetson test 

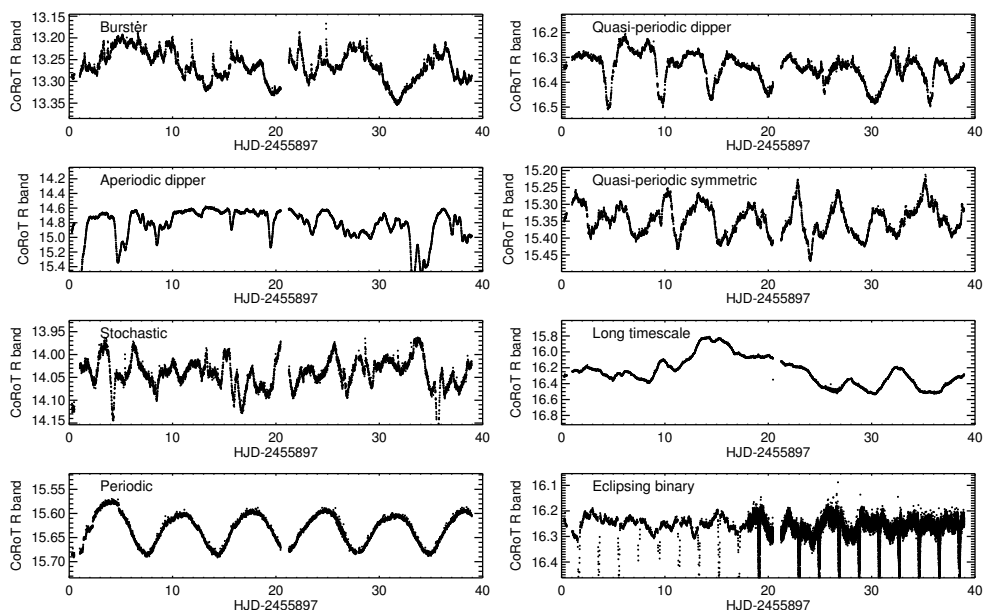

Figure 2. Prototypical examples of the eight optical morphology classes that we identify among CoRoT time series of NGC 2264 members. Each morphology type is labeled on the light curve.

between the IRAC1 and IRAC2 channels are denoted "non-variable." Examples of these classes are shown in the optical in Figure 3, and in the infrared in Figure 4.

To confirm our visual light curve classification, we have developed two statistics (" $Q$ " and " $M$ ") to quantify flux behavior in the optical and infrared bands. We define them in [7], but in brief, they represent the degree of stochasticity $(Q)$ and the degree of flux asymmetry $(M)$ in a light curve. As shown in Figure 5, we find that strongly positive $M$ values accurately select for dipper behavior, while negative $M$ values demarcate bursters. $Q$, on the other hand, represents the strength of the residuals left after a phased periodic trend is subtracted from a light curve. It is close to zero for strongly periodic stars and 1.0 or greater for stochastic stars. Figure 5 confirms that these two statistics are excellent for discriminating the light curve behavior detected by eye.

Table 2. Summary of optical and infrared light curve morphologies for disk bearing stars observed by the CSI 2264 campaign. The fractions of light curves belonging to each class were measured on a 162-object dataset.

Uncertainties on each of these fractions are $2-4 \%$.

\begin{tabular}{ccc}
\hline Morphology class & Optical & Infrared \\
\hline Bursters & $13 \%$ & $5 \%$ \\
Quasi-periodic dippers & $10.5 \%$ & $3 \%$ \\
Aperiodic dippers & $11 \%$ & $1 \%$ \\
Quasi-periodic symmetric & $17 \%$ & $13.5 \%$ \\
Stochastic & $13 \%$ & $6 \%$ \\
Long timescale & $1 \%$ & $30 \%$ \\
Periodic & $4 \%$ & $3.5 \%$ \\
Eclipsing binary & $1 \%$ & $1 \%$ \\
Unclassifiable & $11 \%$ & $28 \%$ \\
Non-variable & $19 \&$ & $9 \%$ \\
\hline
\end{tabular}



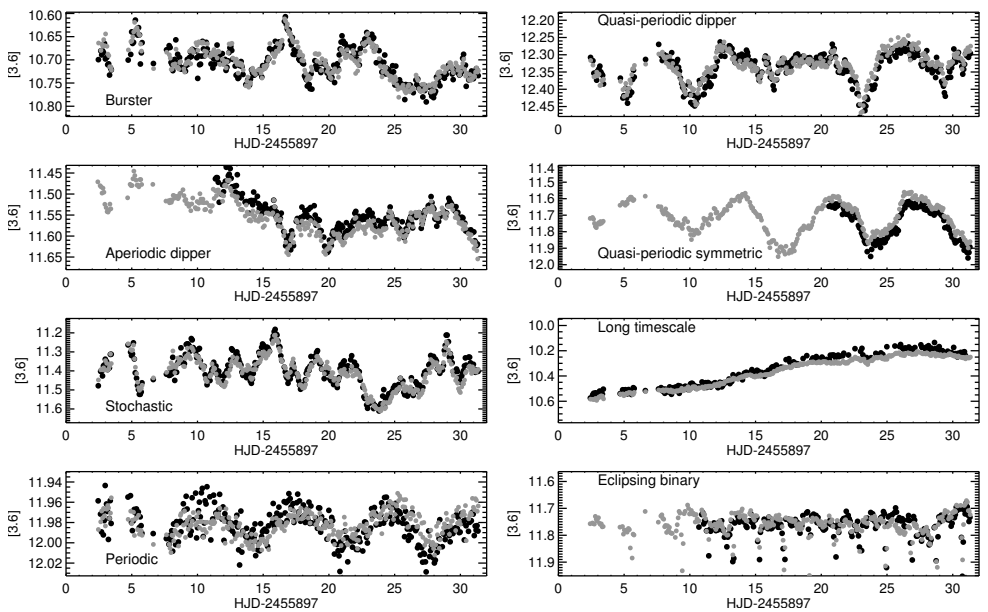

Figure 3. Prototypical examples of the eight infrared morphology classes that we identify among IRAC time series of NGC 2264 members. Black points mark $3.6 \mu \mathrm{m}$ data, and grey points are $4.5 \mu \mathrm{m}$ photometry, with the mean shifted to match that of the $3.5 \mu \mathrm{m}$ light curve. Each morphology type is labeled on the light curve.

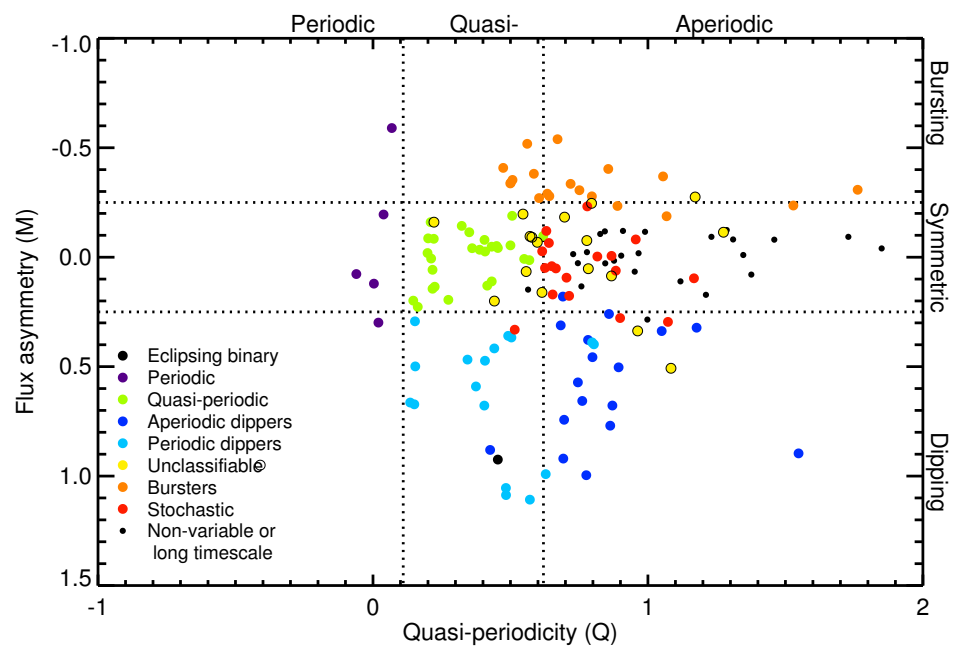

Figure 4. We have identified two statistics that separate the optical light curve behaviors into classes identified by eye, as shown with colored points. These statistics, " $Q$ " and " $M$ ", represent the degree of stochasticity and flux asymmetry, respectively, in the light curve. A small "Q" value represents highly periodic behavior, whereas a value closer to 1.0 indicates that the light curve displays large residuals when phased. The $M$ statistic is negative for light curves dominated by fading events and positive for those with mainly bursting behavior. Adapted from [7]. 
Typically, we classify the CoRoT and Spitzer light curves in different morphological categories. Overall, we find that less than half of our YSOs display well correlated behavior in the optical and infrared bands. This finding suggests that we are witnessing multiple simultaneous variability mechanisms, associated with the star, the accretion streams, and the disk. Further efforts to correlate light curve morphologies in the CSI 2264 dataset with stellar and disk properties will provide insights into the physical origins of variability.

\section{References}

[1] Alencar, S. H. P., Teixeira, P. S., Guimarães, M. M., McGinnis, P. T., Gameiro, J. F., Bouvier, J., Aigrain, S., Flaccomio, E. \& Favata, F., A\&A, 519, 88 (2010)

[2] Baglin, A., Auvergne, M., Barge, P., Deleuil, M., Catala, C., Michel, E., Weiss, W. et al. ESA Special Publication, Vol. 1306, ed. M. Fridlund, A. Baglin, J. Lochard \& L. Conroy, 33 (2006)

[3] Bouvier, J., Chelli, A., Allain, S., Carrasco, L., Costero, R., Cruz-Gonzalez, I., Dougados, C., A\&A, 349, 619 (1999)

[4] Carpenter, J. M., Hillenbrand, L. A. \& Skrutskie, M. F., AJ, 121, 3160 (2001)

[5] Cody, A. \& Hillenbrand, L. H. ApJS, 191, 389 (2010)

[6] Cody, A, Stauffer, J., Micela, G., Baglin, A. \& the CSI 2264 team, AN, 334, 63 (2013)

[7] Cody, A., Stauffer, J., Baglin, A., Micela, G., Rebull, L., Flaccomio, E., Morales-Calderón, M. et al. AJ submitted (2013)

[8] Dahm, S. E. \& Simon, T., AJ, 129, 829 (2005)

[9] Flaherty, K. M. \& Muzerolle, J., ApJ, 719, 1733 (2010)

[10] Gillen, E., Aigrain, S., McQuillan, A., Bouvier, J., Hodgkin, S., Alencar, S. H. P., Terquem, C. et al. A\&A submitted (2013)

[11] Grady, C. A. Muto, T. Hashimoto, J., Fukagawa, M., Currie, T., Biller, B., Thalmann, C. et al. ApJ, 762, 48 (2013)

[12] Herbst, W., Herbst, D. K., Grossman, E. J. \& Weinstein, D., AJ, 108, 1906 (1994)

[13] Joy, A. H., ApJ, 110, 424 (1949)

[14] Lamm, M. H., Bailer-Jones, C. A. L., Mundt, R., Herbst, W., \& Scholz, A., A\&A, 417, 557 (2004)

[15] Makidon, R. B., Rebull, L. M., Strom, S. E., Adams, M. T. \& Patten, B. M., AJ, 127, 2228 (2004)

[16] Morales-Calderón, M., Stauffer, J. R., Rebull, L. M., Plavchan, P., Carpenter, J. M., Whitney, B. A., Covey, K. et al. ApJ, 733, 50 (2011)

[17] Park, B.-G., Sung, H., Bessell, M. S. \& Kang, Y. H., AJ, 120, 894 (2000)

[18] Rebull, L. M., Makidon, R. B., Strom, S. E., Hillenbrand, L. A., Birmingham, A., Patten, B. M., Jones, B. F. et al. AJ, 123, 1528 (2002)

[19] Scholz, A., Xu, X., Jayawardhana, R., Wood, K., Eisloffel, J. \& Quinn, C., MNRAS, 398, 873 (2009)

[20] Stauffer, J. S., Cody, A. M., Alencar, S. H. P., Rebull, L. M., Hillenbrand, L. A., Venuti, L., Turner, N., et al. AJ, submitted (2013)

[21] Sung, H., Bessell, M. S. \& Lee, S.-W., 114, 2644 (1997)

[22] Walker, G., Matthews, J., Kuschnig, R., Johnson, R., Rucinski, S., Pazder, J. \& Burley, G. et al. PASP, 115, 1023 (2003)

[23] Werner, M. W., Roellig, T. L., Low, F. J., Rieke, G., H., Rieke, M., Hoffman, W. F., Young, E. et al. ApJS, 154, 1 (2004) 